\title{
COMPORTAMIENTO DE F'C, MÓDULO DE ELASTICIDAD Y NO. DE REBOTE DE CONCRETOS ECOLÓGICOS TERNARIOS BASE CBCA-HS EXPUESTOS A MGSO4
}

\author{
D. L. GARCÍA GARCÍA ${ }^{1}$, J. L. PÉREZ-DÍAZ², C. GAONA-TIBURCIO ${ }^{3}$, \\ F. ALMERAYA-CALDERÓN ${ }^{3}$, F. J. OLGUIN-COCA ${ }^{4}$, L. D. LÓPEZ-LEÓN ${ }^{4}$, \\ R. VILLEGAS-APAEZ ${ }^{5}$, O. M. LÓPEZ YZA ${ }^{1}$, S. MÁRQUEZ-MONTERO $^{1}$, \\ M. A. BALTAZAR-ZAMORA ${ }^{1}$ \\ ${ }^{1}$ Universidad Veracruzana, Facultad de Ingeniería Civil-Xalapa, Circuito G. Aguirre Beltrán S/N, \\ Lomas del Estadio, Xalapa, Veracruz, México, C.P 91000 \\ ${ }^{2}$ Universidad Autónoma de Chiapas, Facultad de Ingeniería, Tuxtla Gutiérrez, Chiapas, México \\ ${ }^{3}$ Universidad Autónoma de Nuevo León, FIME - CIIIA., San Nicolás de los Garza, N.L, México, C.P 66451 \\ ${ }^{4}$ Universidad Autónoma del Estado de Hidalgo, Mineral de la Reforma, Hidalgo, México \\ ${ }^{5}$ Ingenio Mahuixtlán S.A de C.V, Loc., Mahuixtlán, Coatepec, Veracruz, México
}

\begin{abstract}
RESUMEN
En el presente trabajo de investigación se diseñaron cuatro mezclas de concreto de acuerdo con el método ACI 211.1, una mezcla control 100\% de CPC y tres restantes con sustitución del CPC por combinaciones de Ceniza de Bagazo de Caña de Azúcar y Humo de Sílice (CBCA-HS) en 10\%, 20\% y 30\%. Los ensayos realizados fueron F'c, Módulo de Elasticidad y No. de Rebote, se realizaron acorde a la normativa ONNCCE, los especímenes de estudio se expusieron en agua potable, como medio control y solución al $3.5 \%$ de $\mathrm{MgSO}_{4}$ como medio agresivo por un periodo de 28 días. Los resultados obtenidos indican un buen desempeño de los concretos ecológicos a base de CBCA-HS en comparación con la mezcla control, en particular los concretos ecológicos ternarios con $10 \%$ y $20 \%$ de sustitución.
\end{abstract}

Palabras clave: Concretos ecológicos, F'c., Módulo de Elasticidad, No. de Rebote, CBCA, HS, MgSO 4 .

\begin{abstract}
In the present research work, four concrete mixtures were designed according to the ACI 211.1 method, a 100\% CPC control mixture and three remaining ones with CPC substitution by Sugar Cane Bagasse Ash and Silica Fume (SCBA-SF) combinations in 10\%, 20\% and 30\%. The tests carried out were F'c, Modulus of Elasticity and No. rebound, were carried out according to the ONNCCE standars, the study specimens were exposed in drinking water, as a control medium and solution to $3.5 \%$ of $\mathrm{MgSO}_{4}$ as an aggressive medium for a 28 days period. The obtained results indicate a good performance of the ecological concretes based on CBCA-HS in comparison with the control mixture, in particular the ternary ecological concretes with $10 \%$ and $20 \%$ substitution.
\end{abstract}

Keywords: Ecological concrete; F'c., Elasticity Module, Bounce No., SCBA, SF, $\mathrm{MgSO}_{4}$.

\section{RESUMO}

No presente trabalho de pesquisa, quatro misturas de concreto foram projetadas de acordo com o método ACI 211.1, uma mistura de controle 100\% CPC e três remanescentes com substituição de CPC por combinações de Cinza de bagaço de cana-de-açúcar e fumaça de sílica (CBCA-FS) em 10\%, 20\% e 30\%. Os testes realizados foram F'c, Módulo de Elasticida e Bounce No., foram realizados de acordo com os regulamentos ONNCCE, os espécimes do estudo foram expostos em água potável, como meio de controle e solução a $3,5 \%$ de $\mathrm{MgSO}_{4}$ como meio agressivo para um Período de 28 dias. Os resultados obtidos indicam um bom desempenho dos concretos ecológicos baseados no CBCA-HS em comparação com a mistura controle, em particular os concretos ternários ecológicos com $10 \%$ e $20 \%$ de substituição.

Palavras-chave: Concreto Ecológico, F’c, Módulo de Elasticidade, Bounce No., CBCA, FS, MgSO 4 . 


\section{INTRODUCCIÓN}

El concreto hidráulico es el material de construcción más utilizado a nivel mundial, debido principalmente a sus propiedades físicas, mecánicas, durabilidad, etc., y que en conjunto con el acero de refuerzo se pueden construir Obras Civiles indispensables para el desarrollo de nuestras sociedades (Santiago, G. et al., 2016; García D.L, 2019, Baltazar et.al., 2016, Troconis O.; et al. (2016),, como lo son puentes, edificios, pavimentos, presas, sin embargo, el problema de la corrosión de acero de refuerzo es la principal causa del deterioro prematuro de dicha infraestructura, siendo un problema de miles de millones de dólares ya que la corrosión es un proceso inevitable en los metales (Volpi, V. et al., 2017)a su vez que la producción del Cemento Portland (CP) genera entre el 5\% y $8 \%$ de emisiones de dióxido de carbono al ambiente. Por lo cual se han tratado de encontrar soluciones que retarden o disminuyan el proceso de corrosión y mitiguen las emisiones producidas por la industria cementera (Santiago G. et al., 2016, Landa A. et al., 2019, Landa A. et al., 2018). En el presente trabajo se utilizó la Ceniza de Bagazo de Caña de Azúcar (CBCA) y el Humo de Sílice (HS) como materiales alternativos al CPC debido a sus características puzolánicas (Cordeiro, G.C. et al., 2008, Landa A. et al., 2019, Landa A. et al., 2018), como sustitutos parciales del CPC, con la finalidad de generar una disminución de $\mathrm{CO}_{2}$ y obtener mejoras en las propiedades del concreto para mejorar su desempeño (Landa A. et al., 2018, García D.L, 2019, Ruíz M. et. al.; 2019). Se diseñaron cuatro mezclas de concreto de acuerdo con el ACI 211.1, siendo la primera con el 100\% de CPC y las tres restantes se elaboraron con sustituciones parcialmente en porcentajes de 10\%, 20\% y 30\% del CPC por combinaciones de CBCA y HS posterior a su realización se colocaron en dos diferentes medios, medio control (agua potable) y una solución al 3.5\% de $\mathrm{MgSO}_{4}$ y se realizaron ensayos de f'c, módulo de elasticidad y número de rebote a 7,14 y 28 días.

\section{METODOLOGÍA EXPERIMENTAL}

\subsection{Dosificación y proporcionamiento de las mezclas de concreto}

La dosificación se realizó de acuerdo con el método del ACI 211.1 (ACI, 2004), el cual toma en cuenta principalmente la resistencia a compresión ( $\left.f^{\prime} c\right)$, el revenimiento (trabajabilidad o consistencia) tamaña máximo de agregado, además de la caracterización de las propiedades físicas de los agregados finos y gruesos a emplear (Ariza H.A. et al., 2019, Landa A.E. et al., 2018).

Se llevó a cabo la determinación las propiedades físicas del Agregado Grueso y Agregado Fino que se utilizaron para la elaboración de las mezclas de concreto, los ensayos se realizaron de acuerdo con las normas ONNCCE, y los resultados se muestran en la Tabla 1 (ONNCCE, 2010; ONNCCE, 2010; ONNCCE,2002; ASTM,2008).

Tabla 1. Resultados de la caracterización de los agregados

\begin{tabular}{lcc}
\hline Propiedades físicas de los materiales & Agregado Grueso & Agregado Fino \\
\hline Masa especifica seca (MES)gr/cm & 2.6 & 2.2 \\
Masa Volumétrica Varillada (MVV) Kg/cm & 1433 & 1695 \\
Absorción (A) \% & 1.7 & 1.8 \\
Módulo de Finura (MF) & - & 2.94 \\
Tamaño Máximo Nominal (TMN) & $3 / 4$ & - \\
\hline
\end{tabular}

El cemento portland que se utilizó para realizar las mezclas de concreto fue tipo CPC 30R. Se elaboraron cuatro mezclas de concreto hidráulico, la primera se denominó mezcla control, la cual se realizó con el 100\% de CPC 30R y las tres mezclas restantes se elaboraron con sustituciones parciales 
en 10\%, 20\% y 30\% del CPC 30R por CBCA-HS. En la tabla 2 se muestra la dosificación que se utilizó para cada mezcla.

Tabla 2. Proporcionamiento de las mezclas de concreto para $1 \mathrm{~m}^{3}$

\begin{tabular}{ccccc}
\hline Materiales & $\mathbf{1 0 0 \%}$ CPC & $\begin{array}{c}\text { Sustitución } \\
\text { 10\% CBCA-HS }\end{array}$ & $\begin{array}{c}\text { Sustitución } \\
\text { 20\% CBCA-HS }\end{array}$ & $\begin{array}{c}\text { Sustitución } \\
\text { 30\% CBCA-HS }\end{array}$ \\
\hline Agua & 197.80 & 200.60 & 203.16 & 206.71 \\
Cemento & 314.61 & 284.05 & 252.51 & 220.96 \\
CBCA & 0 & 15.78 & 31.56 & 47.36 \\
HS & 0 & 15.78 & 31.56 & 47.36 \\
Agregado Grueso & 886.00 & 888.64 & 884.22 & 880.73 \\
Agregado Fino & 770.11 & 770.76 & 772.64 & 772.56 \\
\hline
\end{tabular}

\subsection{Caracterización del Concreto Fresco}

De acuerdo a los ensayos de la normativa ONNCCE y ASTM, se determinaron las características del concreto en estado fresco, se realizaron los ensayos de Revenimiento, Temperatura y Masa Volumétrica (ONNCCE, 2010), obteniendo los resultados que se muestran en la tabla 3.

Tabla 4. Propiedades físicas de los concretos empleados.

\begin{tabular}{lcccc}
\hline \multicolumn{1}{c}{ Parámetro } & $\mathbf{1 0 0 \%} \mathbf{C P C}$ & $\begin{array}{c}\text { Sustitución } \\
\text { (0\% CBCA-HS }\end{array}$ & $\begin{array}{c}\text { Sustitución } \\
\mathbf{2 0 \%} \text { CBCA-HS }\end{array}$ & $\begin{array}{c}\text { Sustitución } \\
\text { 30\% CBCA-HS }\end{array}$ \\
\hline Revenimiento & $7.0 \mathrm{~cm}$ & $6.0 \mathrm{~cm}$ & $5.5 \mathrm{~cm}$ & $5.0 \mathrm{~cm}$ \\
Temperatura & $24.0^{\circ} \mathrm{C}$ & $23.5^{\circ} \mathrm{C}$ & $23.5^{\circ} \mathrm{C}$ & $22.5^{\circ} \mathrm{C}$ \\
Masa volumétrica & $2345.83 \mathrm{~kg} / \mathrm{m}^{3}$ & $2307.29 \mathrm{~kg} / \mathrm{m}^{3}$ & $2301.04 \mathrm{~kg} / \mathrm{m}^{3}$ & $2276.04 \mathrm{~kg} / \mathrm{m}^{3}$ \\
\hline
\end{tabular}

\subsection{Ensayos en concreto endurecido}

Los ensayos que se realizaron a los concretos ecológicos en estado endurecido fueron de acuerdo con la normativa ONNCCE (ONNCCE, 2006; ONNCCE, 2013; ONNCCE, 2014), el nombre del ensayo y la norma específica se presentan en la tabla 4.

Tabla 4. Ensayos a concretos ecológicos en estado endurecido.

\begin{tabular}{lc}
\hline \multicolumn{1}{c}{ Ensayo } & \multicolumn{1}{c}{ Norma } \\
\hline Resistencia a la compresión de especímenes & NMX-C-183-ONNCCE-2014 \\
Determinación de número de rebote & NMX-C-192-ONNCCE-2006 \\
Módulo de elasticidad estático & NMX-C-128-ONNCCE-2013 \\
\hline
\end{tabular}

\subsection{Características y nomenclatura de los especímenes de prueba}

Se realizaron especímenes por cada mezcla de concreto y se colocaron en dos diferentes medios, a los cuales se les denoto la siguiente nomenclatura establecida en la tabla 5.

Tabla 5. Nomenclatura de especímenes

\begin{tabular}{ccc}
\hline Mezcla & Medios de exposición \\
\hline MC & & \\
M10 & 1 & 2 \\
M20 & & \\
M30 & & \\
\hline
\end{tabular}

- $\quad$ MC; Mezcla control elaborada con 100\% CPC 30R

- M10: Mezcla elaborada con 90\% CPC, 5\% CBCA y 5\% HS 
- M20: Mezcla elaborada con $80 \%$ CPC, $10 \%$ CBCA y $10 \%$ HS

- M30: Mezcla elaborada con $70 \%$ CPC, $15 \%$ CBCA y $15 \%$ HS

- Medio de contacto: Agua Potable (1) o Sulfato de magnesio (2)

\section{RESULTADOS}

\subsection{Resistencia a la compresión}

En la figura 1 se muestran los resultados de resistencia a la compresión de las cuatro mezclas de estudio, una de concreto normal (100\% CPC 30R) y las tres restantes de concretos ecológicos a base de sustitución parcial del CPC 30R por combinaciones de CBCA-HS en 10, 20 y 30\%, los ensayos fueron a $\operatorname{los}$ 7, 14 y 28 días como lo indica la NMX-C-083-ONNCCE-2002 (ONNCCE, 2002), en dicho periodo los especímenes estuvieron inmersos en el medio control (agua) y en el medio agresivo (solución al 3.5\% de $\mathrm{MgSO}_{4}$ ).

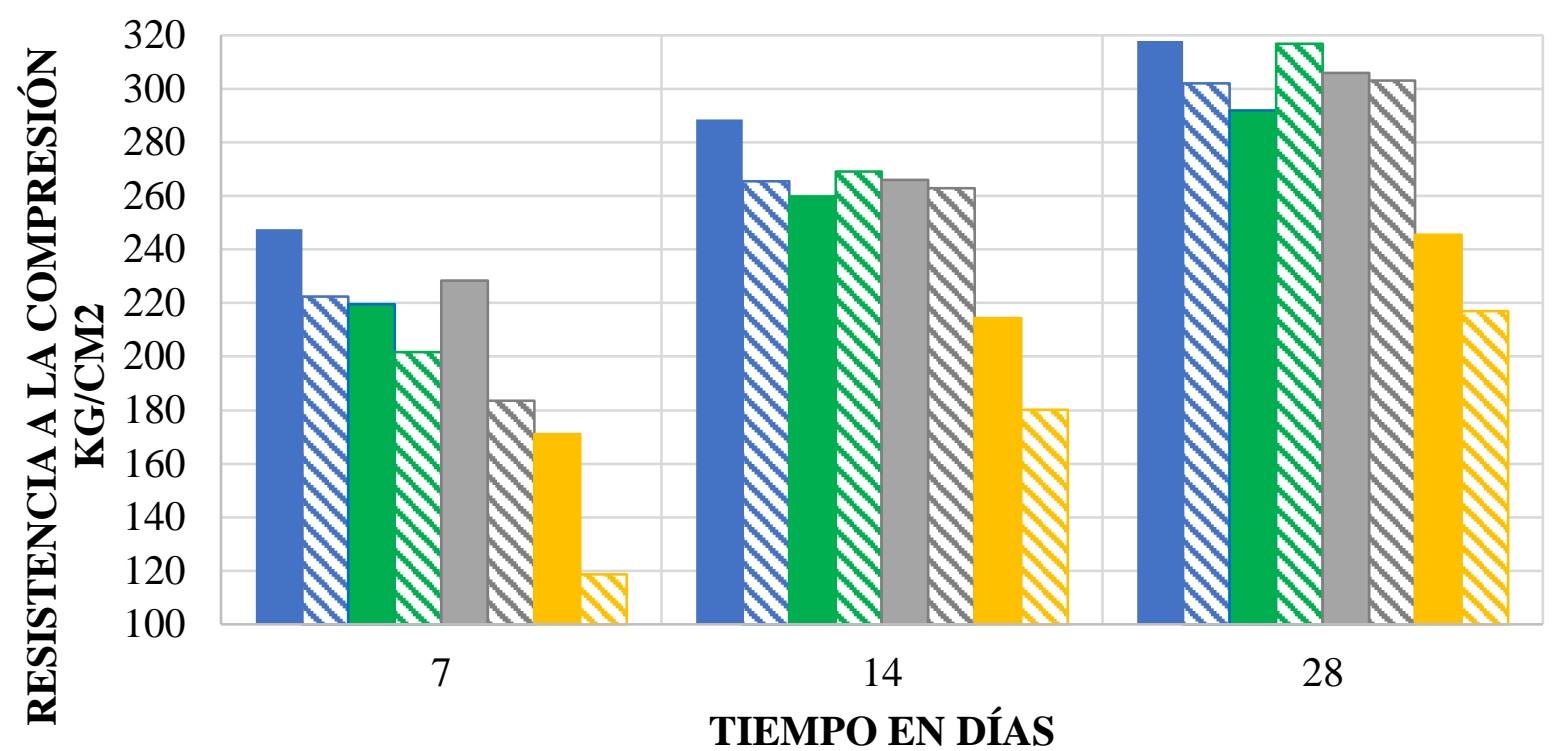

$\square$ MC-1 $₫$ MC-2 $\square$ M10-1 $₫$ M10-2 $\square$ M20-1 $₫$ M20-2 $\square$ M30-1 $₫$ M30-2

Figura 1. F'c de los especímenes expuestos en $\mathrm{H}_{2} \mathrm{O}$ y $\mathrm{MgSO}_{4}$

Al realizar el análisis de los resultados observamos que todas las mezclas presentan un incremento en la resistencia a la compresión, incremento en el F'c, con el paso del tiempo. El espécimen MC-1 presenta el mejor desempeño con los valores mayores en comparación de las demás mezclas, obteniendo a los siete días de curado $247.50 \mathrm{~kg} / \mathrm{cm}^{2}$, aumentando a $288.47 \mathrm{~kg} / \mathrm{cm}^{2}$ el día 14 , llegando a los 28 días a $317.84 \mathrm{~kg} / \mathrm{cm}^{2}$. Por otra parte, la MC-2 presenta un F'c de $222.53 \mathrm{~kg} / \mathrm{cm}^{2}$ en los primeros 7 días y posteriormente logra un valor de $265.40 \mathrm{~kg} / \mathrm{cm}^{2}$ y finaliza a los 28 días con un F'c de 302.16 $\mathrm{kg} / \mathrm{cm}^{2}$, observándose el efecto del medio agresivo en una pequeña disminución en la mezcla MC-2, concreto con $100 \%$ de CPC 30R, pero alcanzando una resistencia por arriba de lo esperado para una relación agua/cemento $=0.62$, las dos mezclas con $100 \% \mathrm{CPC}$, concreto normal presentan en F'c el mejor desempeño. Por lo que respecta a las mezclas concreto ecológicas se tienen resultados muy favorables en las mezclas M10-1, M10-2, M20-1 y M20-2, con un F'c a los 7 días de $219.54 \mathrm{~kg} / \mathrm{cm}^{2}$, $201.57 \mathrm{~kg} / \mathrm{cm}^{2}, 228.51 \mathrm{~kg} / \mathrm{cm}^{2}$ y $183.39 \mathrm{~kg} / \mathrm{cm}^{2}$ respectivamente, para reportar a los 28 días, en el mismo orden F'c de $291.84 \mathrm{~kg} / \mathrm{cm}^{2}, 316.92 \mathrm{~kg} / \mathrm{cm}^{2}, 305.91 \mathrm{~kg} / \mathrm{cm}^{2}$ y $303.16 \mathrm{~kg} / \mathrm{cm}^{2}$. El concreto ecológico identificado como M30 presento el desempeño más bajo, con valores a los 28 días de 245.44 
$\mathrm{kg} / \mathrm{cm}^{2}$ y $217.08 \mathrm{~kg} / \mathrm{cm}^{2}$, para el agua potable y el medio agresivo de sulfato de magnesio. Aun así ambos cumplen la resistencia para ser utilizados en elementos de carga y soporte como columnas y trabes de cualquier obra civil, lo que significa que hasta el concreto con 30\% de sustitución del CPC por residuos agroindustriales e industriales (CBCA-HS) es factible utilizarlos tomando en cuenta la resistencia mecánica obtenida en el ensayo a compresión, $F^{\prime} c$, que es el valor que se utiliza en el diseño de los elementos de las obras construidas a base de concreto reforzado hasta la fecha, la menos en México.

\subsection{Módulo de elasticidad}

Se realizó el ensayo de Módulo de Elasticidad en especímenes de las 4 mezclas de concreto elaboradas para la presente investigación, una como concreto normal y las tres restantes consideradas concretos ecológicos por la sustitución de CPC por combinaciones de CBCA-HS, el ensayo se realizó a una edad de 28 días, de acuerdo con la norma NMX-C-128-ONNCCE-2013 (ONNCCE, 2013), obteniendo los resultados que se muestra en la figura 2.

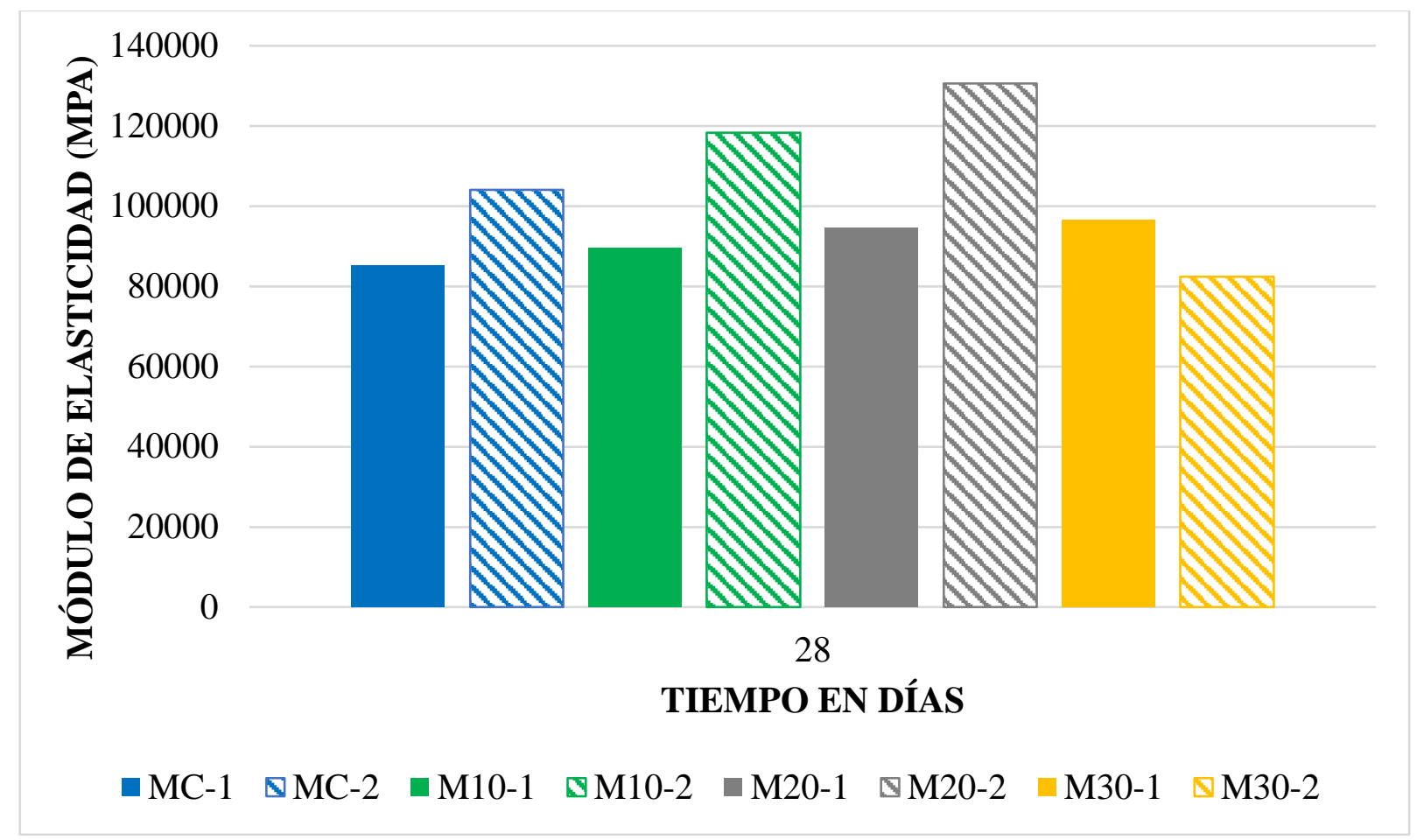

Figura 2. Módulo de elasticidad especímenes expuestos en $\mathrm{H}_{2} \mathrm{O}$ y $\mathrm{MgSO}_{4}$

En el grafico anterior se pueden observar los resultados de las diferentes mezclas de concreto ensayadas a la edad de 28 días, se observa como las mezclas MC-2, M10-2, M20-2, expuestas al medio agresivo presentaron un mejor desempeño que las mezclas expuestas o inmersas en agua potable, y se puede identificar que en el medio control se presenta también un mejor desempeño en el Módulo de Elasticidad por parte de las tres mezclas de concreto ecológico, M10-1, M20-1, M30-1, teniendo las tres valores mayores que la mezcla control MC-1. Se concluye que en el ensayo de Módulo de Elasticidad las mezclas de concreto ecológicos (10, 20 y 30\% de CBCA-HS), presentan mejor desempeño que el concreto convencional o normal, elaborado con $100 \%$ de CPC.

\subsection{No. de Rebote}

Se realizo el ensayo de número de rebote en cada espécimen de las diferentes mezclas de concreto de acuerdo con la normativa NMX-C-192-ONNCCE-2006 (ONNCCE, 2006) en una edad de 7, 14 y 28 días, obteniendo los resultados que se muestran en la figura 3. Se observa como las mezclas de concreto 
convencional (100\% CPC 30R) y las mezclas de concreto ecológico presentan un comportamiento en el ensayo de No. de Rebote muy similar a los resultados presentados en la Figura 1, teniendo menores resistencias los concretos ecológicos base CBCA-HS.

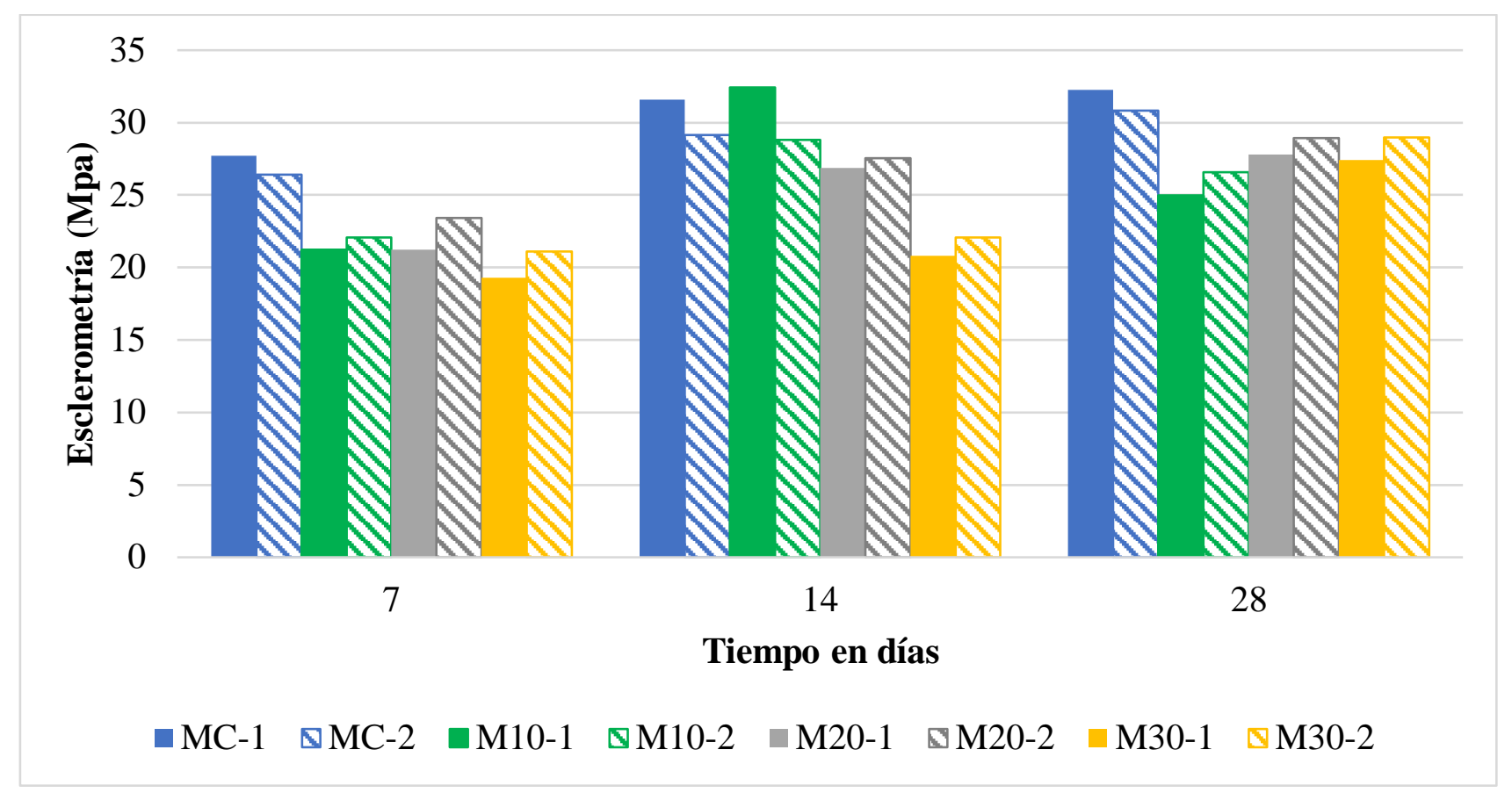

Figura 3. Número de rebote especímenes expuestos en $\mathrm{H}_{2} \mathrm{O}$ y $\mathrm{MgSO}_{4}$

\section{CONCLUSIONES}

En lo que respecta al ensayo de resistencia a compresión el concreto convencional (100\% CPC 30R), presenta resistencias acordes al diseño de la mezcla, con un $\mathrm{F}^{\prime} \mathrm{c}$ a los 28 días que supera el esperado para una relación agua/cemento $=0.62$.

Por lo que respecta a las mezclas concreto-ecológicas se tienen resultados muy favorables en las mezclas M10-1, M10-2, M20-1 y M20-2, con un F'c a los 28 días, de $291.84 \mathrm{~kg} / \mathrm{cm} 2,316.92 \mathrm{~kg} / \mathrm{cm} 2$, $305.91 \mathrm{~kg} / \mathrm{cm} 2$ y $303.16 \mathrm{~kg} / \mathrm{cm} 2$ respectivamente, siendo valores muy cercanos a la mezcla control MC-1. Con sólo una diferencia del $6 \%$ del concreto ecológico que obtuvo la menor resistencia a los 28 días M10-1, con respecto a la mezcla de concreto convencional que obtuvo la mayor resistencia MC-1. En el ensayo de Módulo de Elasticidad de las mezclas de concreto ecológicos (10, 20 y 30\% de CBCAHS), todas presentan mejor desempeño que el concreto convencional o normal, elaborado con $100 \%$ de CPC.

De acuerdo a los resultados obtenidos todas las mezclas de concreto ecológico M10, M20 y M30 analizadas en el presente estudio, cumplen con las propiedades mecánicas, F'c, Modulo de Elasticidad y No. de Rebote para ser utilizados en elementos de carga y soporte como columnas y trabes de cualquier obra civil, lo que significa que hasta el concreto con $30 \%$ de sustitución del CPC por residuos agroindustriales e industriales (CBCA-HS) es factible utilizarlos para la infraestructura civil. Se recomienda a pesar de los resultados mecánicos favorables que se obtuvieron realizar evaluaciones de Durabilidad en el caso de medios agresivos en donde se pudieran construir dichas obras civiles. 


\section{AGRADECIMIENTOS}

M.A. Baltazar-Zamora, et. al., agradecen a PRODEP por el Apoyo otorgado por la SEP, al Cuerpo Académico UV-CA-458 "Sustentabilidad y Durabilidad de Materiales para la Infraestructura Civil", en el marco de la Convocatoria 2018 para Fortalecimiento de Cuerpos Académicos con IDCA 28593.

\section{REFERENCIAS}

Ariza H.A., Croche R., Gaona C., Almeraya F., Ramírez A.C., Villegas R., Márquez S., BaltazarZamora M.A. (2019). "Evaluación de la corrosión del acero galvanizado y acero AISI 304 embebidos en concretos ecológicos con sustitución de CPC por 10, 20 y 30\% de CBCA-HS expuestos a $\mathrm{Na}_{2} \mathrm{SO}_{4}$ al 3.5\%." Memorias del XXXIV Congreso Nacional de la Sociedad Mexicana de Electroquímica, 12th Meeting of the Mexican Section of the Electrochemical Society, SMEQ, Querétaro (México).

ASTM International. (2008), ASTM C1064/C1064M - 08 Standard test method for temperature of freshly Mixed Hydraulic-Cement Concrete

Baltazar-Zamora M.A., Santiago, G., Moreno V., Croche R., De la Garza M., Estupiñan, F., Zambrano, P., Gaona, C. (2016). Electrochemical Behaviour of Galvanized Steel Embedded in Concrete Exposed to Sand Contaminated with $\mathrm{NaCl}$. Int. J. Electrochem. Sci. 11, 10306-10319, doi: 10.20964/2016.12.28. Instituto Mexicano del Cemento y del Concreto, A. C. (2004) "Proporcionamiento de Mezclas, Concreto Normal, Pesado y Masivo ACI 211.1", IMCYC, México, D.F. American Concrete Institute 2002 (ACI 211.1-91).

Cordeiro, G.C., Toledo, R.D., Tavares, E.R., Fairbairn E.M. (2008), Pozzolanic activity and filler effect of sugar cane bagasse ash in Portland cement and lime mortars. Cement and Concrete Composites. 30(5):410-418

García D.L, Cabral J.A, Gaona C., Almeraya F., Ramírez A.C., Croche R., Baltazar-Zamora M.A. (2019) "Influencia del medio ambiente de exposición y tipo de curado en la cinética de corrosión de concreto reforzado" Memorias del XXXIV Congreso Nacional de la Sociedad Mexicana de Electroquímica, 12th Meeting of the Mexican Section of the Electrochemical Society, SMEQ, Querétaro (México).

García D.L, Cabral J.A, Gaona C., Almeraya F., Ramírez A.C., Villegas R., Marquez S., BaltazarZamora M.A. (2019). "Eficiencia anticorrosiva del acero AISI 304 como refuerzo en concretos ecológicos ternarios base CBCA-HS expuestos a sulfato de magnesio". Memorias del XXXIV Congreso Nacional de la Sociedad Mexicana de Electroquímica, 12th Meeting of the Mexican Section of the Electrochemical Society, SMEQ, Querétaro (México).

Landa A., Gaona C., Almeraya F., Ramírez A.C., López O. M., Vélez J.A., Solorzano R., Márquez S., Baltazar-Zamora M.A. (2019), "Evaluación electroquímica del acero inoxidable AISI 316 embebido en concretos sustentables base ceniza de bagazo de caña de azúcar expuestos a un medio marino" Memorias del XXXIV Congreso Nacional de la Sociedad Mexicana de Electroquímica, 12th Meeting of the Mexican Section of the Electrochemical Society, SMEQ, Querétaro (México).

Landa A., Landa A., Rangel J.M., Almeraya F., Croche B., Baltazar-Zamora M.A. (2019). "Comportamiento de la corrosión del acero AISI 316 como refuerzo en concretos ternarios sustentables con sustitución parcial de CPC en 10\%, $20 \%$ y 30\% de CBCA-HS inmersos en agua de mar". Memorias del XXXIV Congreso Nacional de la Sociedad Mexicana de Electroquímica, 12th Meeting of the Mexican Section of the Electrochemical Society, SMEQ, Querétaro (México).

Landa A.E., Croche R., López O.M., Galván R., Cabral J.A., Gaona C., Almeraya F., Baltazar-Zamora M.A. (2018). Corrosion Behavior of AISI 316 Stainless Steel As Reinforcement in Ternary Sustainable Concrete Based on SCBA-SF Exposed in Seawater, ECS Meeting Abstracts, MA2018-02 584-584

Landa A.E., Castillo S., Reyes J.A., Villegas R., Galván R., Cabral J.A., Gaona C., Almeraya F., Baltazar-Zamora M.A. (2018). Evaluation of the Corrosion of AISI 304 Stainless Steel Embedded in

D. L. GARCÍA GARCÍA, et al 
Sustainable Concrete with High Volumes of SCBA-SF Exposed in Marine Environment, ECS Meeting abstracts, MA2018-02 587

Landa A.E., Croche R., Márquez S., Villegas R., Ariza H.A., Estupiñan F., Gaona C., Almeraya F., Baltazar-Zamora M.A. (2018). Corrosion Behavior 304 and 316 Stainless Steel as Reinforcement in Sustainable Concrete Based on Sugar Cane Bagasse Ash Exposed to $\mathrm{Na}_{2} \mathrm{SO}_{4}$, ECS transaction, 84, 179188.

Landa A.E., Croche R., Márquez S., Galván R., Gaona C., Almeraya F., Baltazar-Zamora M.A. (2018). Correlation of Compression Resistance and Rupture Module of a Concrete of Ratio $w / c=0.50$ with the Corrosion Potential, Electrical Resistivity and Ultrasonic Pulse Speed, ECS, 84, 217-227

Organismo Nacional de Normalización y Certificación de la Construcción y Edificación (2002), "NMXC-083-ONNCCE-2002: Industria de la Construcción - Concreto - Determinación de la Resistencia a la Compresión de Especímenes - Método de Ensayo", México

Organismo Nacional de Normalización y Certificación de la Construcción y Edificación, (2006), "NMX-C-192-ONNCCE-2006: Industria de la Construcción - Concreto - Determinación del Número de Rebote Utilizando el Dispositivo Conocido como Esclerómetro", México

Organismo Nacional de Normalización y Certificación de la Construcción y Edificación (2010), "NMXC-105-ONNCCE-2010: Industria de la Construcción - Concreto Hidráulico Ligero Para uso Estructural - Determinación de la Masa Volumétrica", México

Organismo Nacional de Normalización y Certificación de la Construcción y Edificación (2010), "NMXC-156-ONNCCE-2010: Industria de la Construcción - Concreto Hidráulico - Determinación Del Revenimiento En El Concreto Fresco", México

Organismo Nacional de Normalización y Certificación de la Construcción y Edificación (2013), "NMXC-128-ONNCCE-2013: Industria de la Construcción - Concreto Sometido a Compresión Determinación del Módulo de Elasticidad Estático y Relación de Poisson”, México

Organismo Nacional de Normalización y Certificación de la Construcción y Edificación (2014), "NMXC-183-ONNCCE-2012: Industria de la construcción - Materiales asfálticos - Punto de reblandecimiento en cementos asfálticos (anillo y esfera) - Método de ensayo", México

Ruíz M., Landa A., Cabral J.A., Gaona C., Almeraya F., Malpica R., Márquez S., Baltazar M.A. (2019), "Influencia del tipo de acero en el comportamiento de la corrosión de concretos sustentables ternarios elaborados con ceniza de bagazo de caña de azúcar y humo de sílice expuestos a sulfato de sodio" Memorias del XXXIV Congreso Nacional de la Sociedad Mexicana de Electroquímica, 12th Meeting of the Mexican Section of the Electrochemical Society, SMEQ, Querétaro (México).

Santiago, G., Baltazar-Zamora M.A., Galván, R., López, L.D., Zapata, F., Zambrano, P., A. Gaona, C. and Almeraya, F. (2016), Electrochemical evaluation of reinforcement concrete exposed to soil type sp contaminated with sulphates. Int. J. Electrochem. Sci. 11,4850-4864, doi: 10.20964/2016.06.31

Santiago, G., Baltazar-Zamora M.A., Olguín, J., López, L.D., Galván, R., Ríos, A. Gaona, C. and Almeraya, F. (2016), "Electrochemical evaluation of a stainless steel as reinforcement in sustainable concrete exposed to chlorides". Int. J. Electrochem. Sci. 11, 2994

Troconis de Rincon, O.; et al. (2016), "Reinforced Concrete Durability in Marine Environments DURACON Project: Long-Term Exposure". Corrosion. 72, 824.

Volpi, V., López L.D., Hernández, J., Baltazar, M.A., Olguín, F.J. and López, A.L. (2017), “Corrosion study in reinforced concrete made with mine waste as mineral additive”. Int. J. Electrochem. Sci. 12,2231, doi: 10.20964/2017.01.08. 